\title{
Phase 2 study of bosutinib in Japanese patients with newly diagnosed chronic phase chronic myeloid leukemia
}

Masayuki Hino, Itaru Matsumura, Shin Fujisawa, Kenichi Ishizawa, Takaaki Ono, Emiko Sakaida, Naohiro Sekiguchi, Yusuke Tanetsugu, Kei Fukuhara, Masayuki Ohkura, Yuichiro Koide, Naoto Takahashi

\begin{tabular}{|c|c|}
\hline Citation & International Journal of Hematology. 112(1); 24-32 \\
\hline Issue Date & 2020-07 \\
\hline Published & 2020-04-11 \\
\hline Type & Journal Article \\
\hline Textversion & Author \\
\hline $\begin{array}{c}\text { Electronic } \\
\text { supplementary } \\
\text { material }\end{array}$ & $\begin{array}{l}\text { Electronic supplementary material is available at } \\
\text { https://doi.org/10.1007/s12185-020-02878-x. }\end{array}$ \\
\hline Relation & $\begin{array}{l}\text { (C) The Japanese Society of Hematology. This is a post-peer-review, pre-copyedit } \\
\text { version of an article published in International Journal of Hematology. The } \\
\text { final authenticated version is available online at: } \\
\text { https://doi.org/10.1007/s12185-020-02878-x. } \\
\text { See Springer Nature terms. } \\
\text { https://www.springer.com/gp/open-access/publication-policies/aam-terms-of-use. } \\
\text { C 日本血液学会. この原稿は「私的使用」にかぎり利用できます。その他の利用に } \\
\text { は、著作権者の許可が必要です。http://www.jshem.or.jp/. }\end{array}$ \\
\hline DOI & $10.1007 / \mathrm{s} 12185-020-02878-x$ \\
\hline
\end{tabular}

Self-Archiving by Author(s)

Placed on: Osaka City University Repository

Hino, M., Matsumura, I., Fujisawa, S. et al. Phase 2 study of bosutinib in Japanese patients with newly diagnosed chronic phase chronic myeloid leukemia. International Journal of Hematology. 112, 24-32 (2020). Doi:10.1007/s12185-020-02878-x 
ORIGINAL ARTICLE

Phase 2 study of bosutinib in Japanese patients with newly diagnosed chronic phase chronic myeloid leukemia

Masayuki Hino $^{1} \cdot$ Itaru Matsumura $^{2} \cdot$ Shin Fujisawa ${ }^{3} \cdot$ Kenichi Ishizawa $^{4} \cdot$ Takaaki Ono $^{5}$.

Emiko Sakaida ${ }^{6} \cdot$ Naohiro Sekiguchi $^{7} \cdot$ Yusuke Tanetsugu $^{8} \cdot$ Kei Fukuhara $^{8} \cdot$ Masayuki Ohkura $^{8}$

- Yuichiro Koide ${ }^{8} \cdot$ Naoto Takahashi ${ }^{9}$

${ }^{1}$ Osaka City University Hospital, Osaka, Japan

${ }^{2}$ Kindai University Hospital, Osaka, Japan

${ }^{3}$ Yokohama City University Medical Center, Yokohama, Japan

${ }^{4}$ Yamagata University Hospital, Yamagata, Japan

${ }^{5}$ Hamamatsu University Hospital, Shizuoka, Japan

${ }^{6}$ Chiba University Hospital, Chiba, Japan

${ }^{7}$ National Hospital Organization Disaster Medical Center, Tokyo, Japan

${ }^{8}$ Pfizer R\&D Japan GK, Tokyo, Japan

${ }^{9}$ Akita University Hospital, Akita, Japan

Corresponding author: Masayuki Hino, Department of Hematology Osaka City University

Hospital. 1-4-3, Asahi-machi, Abeno-ku, Osaka, Japan 545-8585. Tel: +81-6-6645-3881; E-mail:

hinom@med.osaka-cu.ac.jp

Running head: Bosutinib for newly diagnosed CP CML in Japan 
Manuscript contents:

Abstract word count: 199/200 words

Text word count: 4445/5600 words

Table/figure count: 5 tables/1 figure

Reference count: 36 


\begin{abstract}
This open-label, single-arm, phase 2 study (ClinicalTrials.gov, NCT03128411) evaluated the efficacy, safety, and pharmacokinetics of bosutinib at a starting dose of $400 \mathrm{mg}$ once daily (QD) in Japanese patients with newly diagnosed chronic phase chronic myeloid leukemia (CP CML). The primary endpoint was major molecular response (MMR) at Month 12 in the modified astreated population (Philadelphia chromosome-positive $[\mathrm{Ph}+]$ patients with e13a2/e14a2 transcripts). Sixty Japanese patients with CP CML were treated with bosutinib; median age was 55 years (range, 20-83), 60.0\% were male, and all were $\mathrm{Ph}+$ and had e13a2/e14a2 transcripts. After median follow-up of 16.6 months (range, 11.1-21.9), 41 (68.3\%) patients remained on bosutinib. The MMR rate at Month 12 was 55.0\% (2-sided 90\% confidence interval: 44.4-65.6). There were no on-treatment transformations to accelerated/blast phase, and no patient died on treatment or within 28 days of the last bosutinib dose. The most common treatment-emergent adverse events were diarrhea $(86.7 \%)$, increased alanine aminotransferase $(55.0 \%)$, and increased aspartate aminotransferase (46.7\%). The primary objective of this phase 2 study was met, and there were no new safety signals for bosutinib. These data suggest bosutinib is an effective first-line treatment option for Japanese patients with newly diagnosed CP CML.
\end{abstract}

Keywords Bosutinib · Tyrosine kinase inhibitor · Chronic myeloid leukemia · Japan 


\section{Introduction}

Bosutinib, a second-generation Src/Abl tyrosine kinase inhibitor (TKI), is approved at a starting dose of $500 \mathrm{mg}$ once daily (QD) in many countries, including Japan, for patients with Philadelphia chromosome-positive $(\mathrm{Ph}+)$ chronic phase $(\mathrm{CP})$, accelerated phase (AP), or blast phase (BP) chronic myeloid leukemia (CML) after prior therapy [1-3]. The indication for bosutinib was expanded as first-line therapy for patients with newly diagnosed CP CML, at a starting dose of $400 \mathrm{mg}$ QD, in 2017 by the US Food and Drug Administration [1] and in 2018 by the European Medicines Agency [2]. Approval of first-line bosutinib for CP CML was based on primary data from the global phase 3 BFORE trial, which compared bosutinib versus imatinib [4]. The primary endpoint of major molecular response (MMR) rate at Month 12 in patients with $\mathrm{Ph}+\mathrm{CP}$ CML and e13a2/e14a2 transcripts was significantly higher with bosutinib versus imatinib (47.2\% vs 36.9\%; 2-sided $P=0.02)$, and complete cytogenetic response rate (CCyR) by Month 12 was $77.2 \%$ versus $66.4 \%$ (2-sided $P=0.0075)$. The most common adverse events (AEs) in bosutinib-treated patients were diarrhea $(70.1 \%)$, nausea $(35.1 \%)$, thrombocytopenia (35.1\%), and increased alanine aminotransferase (ALT; 30.6\%), which were consistent with the known toxicity profile of bosutinib [5-7].

Although the efficacy and safety of first-line bosutinib have been established in a global population [4], regional populations have not been fully investigated. Therefore, we conducted a phase 2 study to evaluate the efficacy, safety, and pharmacokinetics of bosutinib at a starting dose of $400 \mathrm{mg}$ QD in Japanese patients with newly diagnosed CP CML.

\section{Methods}

\subsection{Study design}


This is an ongoing open-label, single-arm, phase 2 study to evaluate the efficacy, safety, and pharmacokinetics of bosutinib in adult Japanese patients with newly diagnosed CP CML. The study was conducted in accordance with the Declaration of Helsinki, the International Conference on Harmonisation Guidelines for Good Clinical Practice, and local regulatory requirements. The study protocol, protocol amendments, and informed consent documents (provided by all patients) were approved by the Institutional Review Board/Ethics Committee at each study center in Japan. The trial is registered on ClinicalTrials.gov (NCT03128411).

\subsection{Patients and treatment}

Patients were $\geq 20$ years of age with a molecular diagnosis of CP CML (detection of $B C R-A B L 1$ rearrangement) within 6 months, Eastern Cooperative Oncology Group (ECOG) performance status 0 or 1, adequate renal and hepatic function, and had resolved acute effects of any prior therapy to baseline severity or grade $\leq 1$. Female patients of childbearing potential were required to have a negative serum pregnancy test at screening. Patients were excluded if they had prior treatment for CML (except for hydroxyurea [hydroxycarbamide] within 6 months); central nervous system involvement; extramedullary disease only; major surgery or radiotherapy within 14 days of registration; history of clinically significant or uncontrolled cardiac disease; active, uncontrolled bacterial, fungal, or viral infection; clinically significant gastrointestinal disorder;

history of another malignancy within 5 years prior to registration (except for basal cell carcinoma or cervical carcinoma in situ or stage $1 / 2$ cancer in complete remission for $\geq 12$ months); or uncontrolled hypomagnesemia or uncorrected hypokalemia.

Patients received bosutinib at a starting dose of $400 \mathrm{mg}$ QD. The dose could be escalated in 100-mg increments to a maximum of $600 \mathrm{mg}$ QD in patients with unsatisfactory response (e.g., BCR-ABL1 transcripts $>10 \%$ and/or $\mathrm{Ph}+$ metaphases $>35 \%$ at Month 3), no grade $3 / 4$ AEs at the 
time of dose escalation, and resolution of all prior grade 3/4 AEs to grade 1/2 and all grade 2 nonhematologic toxicities to grade $\leq 1$. The bosutinib dose could be interrupted or reduced to 300 mg QD and further to a minimum of $200 \mathrm{mg}$ QD (with approval by the study sponsor) to manage toxicities. Bosutinib treatment was to continue for approximately 3 years after registration of the last patient, representing the 12 -month core treatment phase and the following $\geq 24$-month extension phase, or until the end of the study, treatment failure, unacceptable toxicity, death, or withdrawal of consent, whichever occurred first.

\subsection{Assessments}

Molecular response was assessed at a central laboratory by quantitative reverse transcriptase polymerase chain reaction (PCR) using peripheral blood collected at baseline, every 3 months for the first 24 months of treatment, and every 6 months thereafter, and was evaluated on the International Scale (IS) [8]. Cytogenetic response was locally assessed in $\mathrm{Ph}+$ patients using bone marrow aspirate obtained at baseline, every 3 months for the first 24 months of treatment, and every 6 months thereafter until CCyR or MMR was attained, after which bone marrow aspirate was performed only if clinically indicated. Hematologic response was assessed using peripheral blood collected locally and by clinical evaluation of extramedullary disease. In patients with lack of response, suboptimal response, or loss of response, and in patients who completed treatment, mutation analysis was performed by sequencing at a central PCR laboratory using peripheral blood collected for molecular response assessment.

Progression to AP CML was defined as $15-29 \%$ blasts in blood or marrow, >30\% blasts plus promyelocytes in blood or marrow with blasts $<30 \%$, or $\geq 20 \%$ basophils in blood; progression to BP CML was defined as $\geq 30 \%$ blasts in blood or bone marrow or extramedullary blast proliferation, other than in the spleen. Events included in the analysis of on-treatment 
event-free survival (EFS) were death due to any cause, transformation to AP/BP, loss of a CCyR, loss of a complete hematologic response, and doubling of white blood cell count $(\geq 1$ month apart with a second value of $>20 \times 10^{9} / \mathrm{L}$ and maintained in subsequent assessments for $\geq 2$ weeks) in patients not achieving complete hematologic response.

AEs were monitored throughout the study period and graded according to the National Cancer Institute Common Terminology Criteria, v4.03 [9]. A treatment-emergent AE (TEAE) was defined as any AE that first occurred or worsened in severity after the first administration of the study drug through 28 days after the last dose. The frequency and characteristics of TEAEs of special interest were analyzed by selecting Medical Dictionary for Regulatory Activities (MedDRA) system organ class higher-level group terms, higher-level terms, preferred terms, and standardized MedDRA queries to generate TEAE clusters (Online Resource 1).

Blood samples for pharmacokinetic analysis were collected within 3 hours before bosutinib administration on Days 1, 28, 56, and 84 of treatment. Bosutinib concentrations in plasma were assayed (Syneos Health; Princeton, NJ, USA) using a validated, sensitive, and specific highperformance tandem mass spectrometry method.

\subsection{Statistical analysis}

The as-treated population included all patients who received at least one dose of bosutinib. The modified as-treated population was the primary analysis population for efficacy evaluation and included patients who were $\mathrm{Ph}+$, had e13a2/e14a2 transcripts, and received at least one dose of bosutinib. Safety was analyzed in the as-treated population. Continuous variables were summarized using descriptive statistics and categorical variables were summarized using frequencies and percentages. 
The primary endpoint was MMR $(\leq 0.1 \% B C R-A B L 1$ on IS, corresponding to $\geq 3-\log$ reduction from standardized baseline with $\geq 3000 A B L$ transcripts assessed) at Month 12 (Week 48 ) in the modified as-treated population. A total of 60 patients was required in the modified astreated population for the study to have $>82 \%$ power to reject the null hypothesis (MMR rate of $25 \%$ at Month 12, based on historical MMR rates with imatinib $[7,10])$ and accept the alternative hypothesis (MMR rate of $40 \%$ at Month 12, derived from the null MMR rate and the higher MMR rate with bosutinib vs imatinib in the BFORE study [4]) with a 1-sided $\alpha$-level of 5\%. The 2-sided asymptotic $90 \%$ confidence interval (CI) of MMR rate at Month 12 was calculated.

Secondary endpoints included MMR and CCyR $(0 \mathrm{Ph}+$ chromosomes of $\geq 20$ metaphases or MMR; bone marrow aspirates not required once MMR was achieved) by Month 12 (patients are considered responders if MMR or CCyR occurs at or before Month 12), EFS, overall survival, safety, and pharmacokinetics. Exploratory endpoints included MMR at Months 3, 6, and 9; $\mathrm{MR}^{4}(\leq 0.01 \% B C R-A B L 1$ on IS, corresponding to $\geq 4-\log$ reduction from the standardized baseline with $\geq 9800 A B L$ transcripts assessed) and $\mathrm{MR}^{4.5}(\leq 0.0032 \% B C R-A B L 1$ on IS, corresponding to $\geq 4$.5-log reduction from the standardized baseline with $\geq 30,990 A B L$ transcripts assessed) at Months 3, 6, 9, and 12; $\mathrm{MR}^{1}(\leq 10 \%$ BCR-ABL1 on IS, corresponding to $\geq 1-\log$ reduction from the standardized baseline) at Month $3 ; \mathrm{MR}^{2}(\leq 1 \% B C R-A B L 1$ on IS, corresponding to $\geq 2-\log$ reduction from the standardized baseline) at Month 6 ; and time to $\mathrm{MMR}, \mathrm{MR}^{4}, \mathrm{MR}^{4.5}$, and CCyR.

The data for these analyses are from an unlocked trial database with a data cutoff date of March 12, 2019, after a minimum of 12 months (48 weeks) of follow-up of the last enrolled patient. 


\section{Results}

\subsection{Patients and treatment}

In all, 60 Japanese patients with newly diagnosed CP CML were enrolled in the study and treated with bosutinib (i.e., the as-treated population). All patients were $\mathrm{Ph}+$ and had e13a2/e14a2 transcripts and were included in the modified as-treated population analyzed for efficacy. Median age was 55 years (range, 20-83) and 41 (68.3\%) patients were aged $<65$ years; 36 (60.0\%) patients were male and median weight was $59.8 \mathrm{~kg}$ (range, 34.5-102.6; Table 1). A total of $27(45.0 \%), 26(43.3 \%)$, and 7 (11.7\%) patients had low-, intermediate-, and high-risk Sokal scores, respectively; 58 (96.7\%) patients had ECOG performance status 0.

Median duration of follow-up was 16.6 months (range, 11.1-21.9) and median duration of bosutinib treatment was 15.3 months (range, $0.3-21.9$; Table 2). At the data cutoff date, 41 (68.3\%) patients remained on bosutinib; 18 (30.0\%) patients discontinued due to AEs and 1 (1.7\%) due to physician decision. The most common TEAEs leading to discontinuation were increased ALT $(n=6)$ and increased aspartate aminotransferase (AST; $n=5)$. Other TEAEs that led to discontinuation were increased lipase, drug eruption, and erythema multiforme ( $n=2$ each) and thrombocytopenia, drug-induced liver injury, liver disorder, pneumonia, increased hepatic enzyme, increased pancreatic enzymes, decreased neutrophil count, and pleural effusion ( $n=1$ each). There were no bosutinib discontinuations due to lack of efficacy or suboptimal response.

To manage TEAEs, 33 (55.0\%) patients had $\geq 1$ bosutinib dose reduction and 46 (76.7\%)

had $\geq 1$ dose interruption (Table 2). The bosutinib dose was escalated due to insufficient response in $6(10.0 \%)$ patients. Median dose intensity was $354.7 \mathrm{mg} /$ day (range, $95.3-494.1$ ). 


\subsection{Efficacy}

The MMR rate at Month 12 was 55.0\% (2-sided 90\% CI: 44.4-65.6); the test of the null hypothesis was rejected $(1$-sided $P<0.0001)$, and the primary endpoint was met (Table 3$)$. In patients with low-, intermediate-, and high-risk Sokal scores, respectively, the MMR rate (90\% CI) at Month 12 was 51.9\% (36.0-67.7), 61.5\% (45.8-77.2), and 42.9\% (12.1-73.6). The MMR rate by Month 12, which included patients with a response at or before this time point, was 61.7\% (90\% CI: 51.3-72.0), and the CCyR rate by Month 12 was 80.0\% (90\% CI: 71.5-88.5). Deep molecular responses were attained starting at Month 6, with rates (90\% CI) of 31.7\% (21.8-41.5) for $\mathrm{MR}^{4}$ and $21.7 \%$ (12.9-30.4) for $\mathrm{MR}^{4.5}$ at Month 12 (Fig. 1). The $\mathrm{MR}^{1}$ rate at Month 3 was $80.0 \%$ (90\% CI: 71.5-88.5), and the $\mathrm{MR}^{2}$ rate at Month 6 was $66.7 \%$ (90\% CI: 56.7-76.7). Median (range) time to response in patients who achieved the respective response was 24.1 (12.0-60.1) weeks for MMR and 12.2 (11.9-36.1) weeks for CCyR (Online Resources 2 and 3$)$.

The cumulative incidence $(90 \%$ CI) of EFS events at Month 12 was $1.7 \%(0.2-6.4)$, although data for on-treatment EFS were not mature as of the data cutoff date; there were no ontreatment transformations to AP or BP CML. No patient died on treatment or within 28 days of the last dose of bosutinib. One patient (a 48-year-old male) died beyond 28 days of the last dose due to disease progression. This patient initially received bosutinib $400 \mathrm{mg}$ QD but had the dose reduced to $300 \mathrm{mg}$ QD due to grade 3 thrombocytopenia and subsequently discontinued treatment at Day 85 due to recurrence of grade 3 thrombocytopenia, with no molecular response recorded; death occurred on Day 403 of the long-term follow-up period.

Of the 18 patients who had mutation testing at treatment completion, 16 were evaluable; none had a newly detectable mutation. 


\subsection{Safety}

All patients experienced $\geq 1$ TEAE and $45(75.0 \%)$ had $\geq 1$ grade $3 / 4$ TEAE; serious AEs occurred in 14 (23.3\%) patients. TEAEs led to bosutinib dose reduction in 33 (55.0\%) patients, to dose interruption in $42(70.0 \%)$ patients, and to bosutinib discontinuation in $18(30.0 \%)$ patients. The most common TEAEs were diarrhea (86.7\%), increased ALT (55.0\%), and increased AST (46.7\%; Table 4). Grade 3/4 TEAEs reported in $\geq 10 \%$ of patients were increased ALT (33.3\%), increased AST (18.3\%), diarrhea (15.0\%), increased lipase (15.0\%), lymphopenia (13.3\%), and neutropenia (11.7\%). Grade 3/4 TEAEs were more common in patients aged $\geq 65$ versus $<65$ years $(89.5 \%$ vs $68.3 \%$ ), as were TEAEs leading to bosutinib dose reduction (68.4\% vs $48.8 \%$ ), dose interruption ( $89.5 \%$ vs $61.0 \%$ ), and discontinuation ( $47.4 \%$ vs $22.0 \%$ ); the incidence of serious AEs was similar between age groups (26.3\% vs $22.0 \%)$.

We assessed the characteristics of the common TEAEs, diarrhea, increased ALT, and increased AST. Median time to first event of diarrhea was 1 day (range, 1-271). Diarrhea was frequently managed with concomitant medications, in 49 of 52 (94.2\%) patients who experienced diarrhea; 6 (11.5\%) patients had bosutinib dose interruptions and $2(3.8 \%)$ had dose reductions due to diarrhea. All 6 patients who had dose interruptions due to diarrhea had successful bosutinib rechallenge, i.e., they did not have subsequent diarrhea or discontinue due to subsequent or persistent events. Median time to first event of increased ALT was 15 days (range, 1-169). Of 33 patients with increased ALT, 17 (51.5\%) had bosutinib dose interruptions and 13 (39.4\%) had dose reductions; 16 (48.5\%) patients received concomitant medications. All 17 patients who had dose interruptions due to increased ALT had bosutinib rechallenge; 13 (76.5\%) rechallenges were successful. Median time to first event of increased AST was 15 days (range, 1-57). In all, 14 of $28(50.0 \%)$ of patients with increased AST were treated with concomitant 
medications; $10(35.7 \%)$ patients had bosutinib dose interruptions and $8(28.6 \%)$ had dose reductions. Nine of 10 patients with dose interruptions for increased AST had bosutinib rechallenge; $6(66.7 \%)$ rechallenges were successful.

When evaluated according to categories of special interest, the most frequently reported TEAEs of any grade were gastrointestinal (86.7\%), liver function-related $(80.0 \%)$, infection (65.0\%), rash (55.0\%), and myelosuppression (45.0\%; Online Resource 4). The most common grade 3/4 TEAEs of special interest were liver function-related (48.3\%) and myelosuppression (26.7\%). Within the gastrointestinal cluster, all TEAEs were considered related to bosutinib treatment, and the maximum toxicity for most patients was grade $1(30.0 \%)$ or grade $2(41.7 \%)$ events; $9(15.0 \%)$ patients had a maximum toxicity of grade 3 gastrointestinal TEAE. No patient discontinued bosutinib owing to a gastrointestinal TEAE. Of the $48(80.0 \%)$ patients with liver function TEAEs, 47 had events considered bosutinib-related; the maximum toxicity was grade 1 in $16.7 \%$ of patients, grade 2 in $15.0 \%$, grade 3 in $40.0 \%$, and grade 4 in $8.3 \%$. Ten (16.7\%) patients permanently discontinued treatment due to liver function TEAEs, all of which were characterized by or associated with laboratory abnormalities of increased ALT, increased AST, or both; 8 of these 10 patients discontinued bosutinib within 6 months of initiating treatment. In addition, 28 patients temporarily stopped bosutinib because of this TEAE, with 19 rechallenged successfully. There were no cases of Hy's law or permanent liver injury. The incidence of cardiac, vascular, and hypertension TEAEs was low $(5.0 \%, 1.7 \%$, and $1.7 \%$, respectively). Three patients had pericardial effusion; all events were grade 2 and considered related to bosutinib treatment. One patient had grade 1 peripheral coldness, which was not considered related to study drug. One patient had grade 3 treatment-related hypertension, but this patient had preexisting hypertension at the time of starting bosutinib. 
The most common laboratory abnormalities, based on laboratory test values, were increased creatinine (95.0\%), decreased lymphocyte count (91.7\%), increased ALT (85.0\%), increased AST (81.7\%), and decreased hemoglobin (81.7\%; Table 5).

\subsection{Pharmacokinetics}

Bosutinib plasma trough concentrations were stable over time (Online Resources 5 and 6). The mean \pm standard deviation of bosutinib concentration averaged over Days 28, 56, and 84 was $82.7 \pm 48.0 \mathrm{ng} / \mathrm{mL}$. In patients aged $<65$ versus $\geq 65$ years, bosutinib plasma trough concentrations were similar (mean averaged over Days 28, 56, and 84 was 80.3 vs $89.3 \mathrm{ng} / \mathrm{mL}$ ), except at Day 56, where lower mean concentrations were seen in the younger versus older group (79.3 vs $113.6 \mathrm{ng} / \mathrm{mL}$; Online Resources 7 and 8). For other baseline covariates (e.g., sex and body weight), an analysis of covariance model found no differences between subgroups (data not shown).

\section{Discussion}

The primary objective of this phase 2 study of bosutinib in Japanese patients with newly diagnosed CP CML was met, and the safety profile of bosutinib was consistent with previous studies [4-7]. The data reported here suggest bosutinib is an effective first-line treatment option for Japanese patients with newly diagnosed CP CML, in addition to its established role in the second-line or later setting in this regional population.

Our findings extend those of the global phase 3 BFORE trial, which established improved efficacy of bosutinib versus imatinib in patients with newly diagnosed CP CML [4]. In addition to the proportion of Asian patients in the BFORE trial who received bosutinib (12.2\%), differences between the Japanese population analyzed here and the global BFORE population 
should be noted. The proportion of patients aged $\geq 65$ years $(31.7 \%$ vs $19.5 \%)$, with low-risk Sokal scores (45.0\% vs 38.2\%), and with ECOG performance status 0 (96.7\% vs $70.7 \%$ ) was higher in the present phase 2 Japan study versus the bosutinib arm of the BFORE trial [4]. Interestingly, this is in contrast with prior publications that reported a lower age for diagnosis of CML in Asian versus Western patients [11, 12], as well as higher Sokal risk score [13]. Treatment characteristics also differed in the present study versus BFORE. There was, in the present study versus BFORE, a lower median dose intensity ( 354.7 vs $391.8 \mathrm{mg} /$ day), likely due to the higher rate of bosutinib dose reductions (55.0\% vs $34.0 \%)$ and dose interruptions (70.0\% vs $56.7 \%$ ) due to AEs [4].

Notwithstanding the variability in patient and treatment characteristics in this Japanese population versus a global population with newly diagnosed CP CML, the MMR rate at Month $12(55.0 \%)$ was consistent with that reported in the bosutinib arm of the multinational BFORE trial (47.2\%). Other efficacy endpoints were also comparable or more favorable in this phase 2 study versus the bosutinib arm of BFORE, including CCyR rate by Month 12 (80.0\% vs 77.2\%), rates of $\mathrm{MR}^{4}\left(31.7 \%\right.$ vs $20.7 \%$ ) and $\mathrm{MR}^{4.5}$ (21.7\% vs $\left.8.1 \%\right)$ at Month 12 , and time to MMR (24.1 vs 24.7 weeks) and CCyR (12.2 vs 23.9 weeks) [4].

No new safety signals were observed for first-line bosutinib in Japanese patients. The most common individual TEAEs with bosutinib were consistent between the current phase 2 Japan study and the global BFORE trial [4]. The rate of grade 3/4 TEAEs in bosutinib-treated patients was higher in the Japanese population than in the BFORE population (75.0\% vs 56.0\%), although rates of any grade TEAEs (100\% vs $98.1 \%$ ) and serious AEs (21.7\% vs $20.1 \%$ ) were similar. In addition, a greater proportion of patients in this study versus BFORE discontinued bosutinib due to AEs (30.0\% vs 13.8\%); the most common TEAEs leading to bosutinib 
discontinuation in the respective studies were increased ALT (10.0\% vs 4.9\%) and increased AST ( $8.3 \%$ vs $2.2 \%)$. When evaluated according to categories of special interest, liver function (80.0\% vs $39.9 \%)$, infection $(65.0 \%$ vs $44.4 \%)$, and rash TEAEs $(55.0 \%$ vs $33.6 \%)$ occurred at higher rates with bosutinib in Japanese patients compared with the global population of BFORE, as did the rate of grade $3 / 4$ liver function TEAEs (48.3\% vs $24.3 \%$ ). Despite the higher frequency of any-grade and grade 3/4 liver function TEAEs in this study, there were no Hy's law cases, permanent drug-induced liver injuries, or hepatotoxicity-related fatalities. In a prior phase 1/2 study of bosutinib in Japanese patients with CP CML after prior therapy, the incidence of liver function TEAEs was $62 \%$ [14], which prompted a recommendation for more frequent monitoring of liver function, i.e., twice monthly during the first 2 months and once during the third month of bosutinib treatment, in the Japan versus US prescribing information $[1,3]$; physicians may opt for additional monitoring beyond this guidance.

Differences in some safety outcomes in the present phase 2 study versus BFORE may, in part, reflect the higher proportion of patients aged $\geq 65$ years in the phase 2 study. In the current study and in previous analyses of the phase 3 BFORE [15] and BELA [16] trials, the rate of grade 3/4 TEAEs was higher in bosutinib-treated patients aged $\geq 65$ years compared with those aged $<65$ years. In addition, the average bosutinib plasma trough concentration in this Japanese population was $\sim 1.12$-fold higher than that observed in bosutinib-treated patients in the global BFORE trial (Pfizer Inc., data on file). A pharmacokinetic-pharmacodynamic analysis using data from the BELA and phase 1/2 studies of bosutinib at a starting dose of $500 \mathrm{mg}$ QD in patients with newly diagnosed [7] and previously treated CP CML [5, 6], respectively, identified an exposure-response relationship for diarrhea and rash in patients with CML receiving bosutinib, but not for increased ALT or AST [17]. Another study of different bosutinib dosing regimens in 
Japanese patients with CML found that liver dysfunction was more prevalent in patients with higher bosutinib trough concentrations [18]. Noting small patient numbers, relationships were not observed here for bosutinib trough concentrations and either increased ALT or AST (data not shown). It will be of interest to identify factors, including comorbidities and pharmacokinetic parameters, which might have influenced the occurrence of TEAEs in Japanese patientsparticularly increased ALT and AST, which most frequently led to bosutinib discontinuation. An exposure-response analysis to evaluate the relationships between bosutinib concentration and TEAEs or efficacy endpoints using a population pharmacokinetic model based on a combined dataset including this study is now being conducted and will be reported separately.

The safety profile of bosutinib is distinct from other TKIs approved as first-line treatment for CP CML $[4,10,19]$; AE profiles should be considered by physicians and patients when selecting an appropriate treatment. Strategies to manage AEs and allow patients to remain on bosutinib treatment have been reported $[20,21]$ and can be applied to Japanese patients. These include dose reductions and interruptions, as well as initiating treatment at a lower dose and incrementally escalating the dose until the recommended dose is reached. Supportive care, including antidiarrheal or antiemetic medication and increased hydration, can help mitigate gastrointestinal AEs. As noted above, liver function abnormalities during bosutinib treatment are a particular concern for Japanese patients, necessitating more intensive monitoring in this population [3]. Bosutinib dose reductions and interruptions are recommended for patients with liver toxicities [20,21], but concomitant medications, such as steroids or glycyrrhizic acid, have also been administered to manage increased ALT or AST in the multinational phase 1/2 study of bosutinib for previously treated patients with CML [22]. 
The occurrence of cardiovascular events with long-term TKI treatment, although infrequent, has become a concern [23, 24]. A retrospective analysis found that Japanese patients with CML treated with TKIs, in particular nilotinib, were at a higher risk for ischemic heart disease compared with the general Japanese population [25]. In the current study, rates of cardiac, vascular, and hypertension TEAEs were low, but longer follow-up is needed to characterize cardiovascular events in Japanese patients receiving first-line bosutinib. It is recommended that a patient's cardiovascular risk factors are assessed prior to initiating bosutinib therapy to determine if additional monitoring for cardiac, vascular, and hypertension TEAEs is warranted [20, 21].

To date, two second-generation TKIs, dasatinib and nilotinib, have been approved in Japan as first-line CP CML treatment, based on the global phase 3 DASISION [19] and ENESTnd [10] trials, respectively, both of which showed improved efficacy versus the first-generation TKI imatinib. Analyses of the Japanese subgroups in these trials were reported after 24 months [26] and 5 years of follow-up [27] for DASISION and after 12 months [28] and 5 years of follow-up [29] for ENESTnd. Another recent publication based on a 5-year observational study of first-line TKI treatment in Japan reported higher rates of early molecular response (MR ${ }^{1}$ at Month 3$)$ and durable deep molecular response (MR ${ }^{4.5}$ by Month 36) in patients who received dasatinib or nilotinib compared with those administered imatinib [30]. Although cross-trial comparisons are challenging due to differences such as study design and endpoints, the MMR rate at Month 12 was consistent between the current phase 2 Japan study population (55.0\%) and the Japanese subgroups of ENESTnd who received $300 \mathrm{mg}$ or $400 \mathrm{mg}$ nilotinib twice daily (57\% and 50\%, respectively) [28], as was the MMR rate by Month 12 in the current phase 2 Japan study versus the Japanese population treated with dasatinib in DASISION (61.7\% vs 69\%) [26]. The MR ${ }^{4.5}$ 
rate at 12 months appeared higher with bosutinib (21.7\%) than with $300 \mathrm{mg}$ nilotinib (6.7\%) or $400 \mathrm{mg}$ nilotinib (4.2\%) in the Japanese subgroups of ENESTnd [28]. In line with the known profiles of second-generation TKIs [4, 10, 19], fluid retention and effusion TEAEs were more common in dasatinib-treated Japanese patients with CP CML [26] and increased glucose was more frequently reported in nilotinib-treated Japanese patients [28] compared with bosutinibtreated Japanese patients in the current study. Similar to our findings, there were differences in the rates of certain TEAEs with dasatinib and nilotinib in the Japanese subgroup compared with the global population of DASISION and ENESTnd, respectively [26, 28].

This study is limited by the single-arm design with no comparator, small number of patients, and short follow-up period. Nevertheless, an acceptable toxicity profile was observed for bosutinib in Japanese patients with CP CML, and a substantial proportion of patients had deep molecular responses. Since durable deep molecular responses allow some patients to discontinue TKI treatment and attain treatment-free remission [31], the results from this study are promising; longer follow-up is needed to assess if deep molecular responses with bosutinib are maintained in Japanese patients. It should be noted that the possibility for treatment-free remission after discontinuation of bosutinib has not been prospectively assessed in clinical trials, in contrast with other second-generation TKIs [32-36], but it is anticipated that feasibility is not dependent on the administered TKI.

In conclusion, our findings further substantiate the efficacy and safety of first-line bosutinib in a specific regional population, as was also reported in the overall population in the phase 3 BFORE trial [4], and provide support for bosutinib as an effective treatment option for Japanese patients with newly diagnosed CP CML. 


\section{Acknowledgments}

This study was sponsored by Pfizer. Medical writing support was provided by Joanna Bloom, $\mathrm{PhD}$, of Engage Scientific Solutions and was funded by Pfizer.

\section{Research Data Policy/Data Availability}

Upon request, and subject to certain criteria, conditions and exceptions (see https://www.pfizer.com/science/clinical-trials/trial-data-and-results for more information), Pfizer will provide access to individual de-identified participant data from Pfizer-sponsored global interventional clinical studies conducted for medicines, vaccines and medical devices (1) for indications that have been approved in the US and/or EU or (2) in programs that have been terminated (i.e., development for all indications has been discontinued). Pfizer will also consider requests for the protocol, data dictionary, and statistical analysis plan. Data may be requested from Pfizer trials 24 months after study completion. The de-identified participant data will be made available to researchers whose proposals meet the research criteria and other conditions, and for which an exception does not apply, via a secure portal. To gain access, data requestors must enter into a data access agreement with Pfizer.

\section{Conflict of interest}

Masayuki Hino reports research funding from Pfizer, Novartis, and Otsuka, and honoraria from Pfizer, Novartis, Otsuka, and Bristol-Myers Squibb. Itaru Matsumura reports research funding from Pfizer and Otsuka, speakers bureau with Novartis and Bristol-Myers Squibb, and consultancy with Otsuka. Shin Fujisawa reports honoraria and research funding from Novartis, Pfizer, Otsuka, and Bristol-Myers Squibb. Kenichi Ishizawa reports research funding from Pfizer 
and Otsuka and speakers bureau with Novartis and Bristol-Myers Squibb. Takaaki Ono reports honoraria from Celgene, Merck Sharp \& Dohme, Ono, Novartis, Bristol-Myers Squibb, Pfizer, Otsuka, and Takeda and research funding from, Celgene, Merck Sharp \& Dohme, Ono, Kyowa Hakko Kirin, and Chugai. Emiko Sakaida reports research funding from Bristol-Myers Squibb, Chugai, Ono, and Kyowa Kirin. Naohiro Sekiguchi reports research funding from Ono, A2 Healthcare, Astellas, Janssen, Merck Sharp \& Dohme, Otsuka, Pfizer, PPD-SNBL, Sumitomo Dainippon Pharma, Daiichi Sankyo Company, and Bristol-Myers Squibb. Yusuke Tanetsugu, Kei Fukuhara, Masayuki Ohkura, and Yuichiro Koide report employment by Pfizer R\&D Japan G.K. Naoto Takahashi reports research funding and honoraria from Pfizer, Otsuka, and Novartis, and research funding from Chugai, Eizai, Asahikasei, Ono, Kyowahakko-Kirin, and Toyamakagaku, outside the submitted work. 


\section{References}

1. Pfizer Inc. Bosulif® (bosutinib) prescribing information. 2012 (last update: Dec 2017). Available at: https://www.accessdata.fda.gov/drugsatfda_docs/label/2017/203341s0091bl.pdf. Accessed November 11, 2019.

2. European Medicines Agency. Bosulif® (bosutinib) summary of product characteristics. 2013 (last update: Aug 7, 2019). Available at: https://www.ema.europa.eu/en/medicines/human/EPAR/bosulif. Accessed November 11, 2019.

3. Pfizer Japan Inc. Bosulif® (bosutinib hydrate) prescribing information. Japan; 2017.

4. Cortes JE, Gambacorti-Passerini C, Deininger MW, Mauro MJ, Chuah C, Kim DW, et al. Bosutinib versus imatinib for newly diagnosed chronic myeloid leukemia: results from the randomized BFORE trial. J Clin Oncol. 2018; 36:231-7.

5. Cortes JE, Kantarjian HM, Brummendorf TH, Kim DW, Turkina AG, Shen ZX, et al. Safety and efficacy of bosutinib (SKI-606) in chronic phase Philadelphia chromosomepositive chronic myeloid leukemia patients with resistance or intolerance to imatinib. Blood. 2011; 118:4567-76.

6. Khoury HJ, Cortes JE, Kantarjian HM, Gambacorti-Passerini C, Baccarani M, Kim DW, et al. Bosutinib is active in chronic phase chronic myeloid leukemia after imatinib and dasatinib and/or nilotinib therapy failure. Blood. 2012; 119:3403-12.

7. Cortes JE, Kim DW, Kantarjian HM, Brummendorf TH, Dyagil I, Griskevicius L, et al. Bosutinib versus imatinib in newly diagnosed chronic-phase chronic myeloid leukemia: results from the BELA trial. J Clin Oncol. 2012; 30:3486-92. 
8. Hughes T, Deininger M, Hochhaus A, Branford S, Radich J, Kaeda J, et al. Monitoring CML patients responding to treatment with tyrosine kinase inhibitors: review and recommendations for harmonizing current methodology for detecting BCR-ABL transcripts and kinase domain mutations and for expressing results. Blood. 2006; 108:2837.

9. National Cancer Institute. Common Terminology Criteria for Adverse Events (CTCAE) v4.03. 2010 (last update: Jun 14, 2010). Available at: https://evs.nci.nih.gov/ftp1/CTCAE/CTCAE_4.03/CTCAE_4.03_2010-0614_QuickReference_8.5x11.pdf. Accessed November 11, 2019.

10. Saglio G, Kim DW, Issaragrisil S, le Coutre P, Etienne G, Lobo C, et al. Nilotinib versus imatinib for newly diagnosed chronic myeloid leukemia. N Engl J Med. 2010; 362:22519.

11. Au WY, Caguioa PB, Chuah C, Hsu SC, Jootar S, Kim DW, et al. Chronic myeloid leukemia in Asia. Int J Hematol. 2009; 89:14-23.

12. Kim DW, Banavali SD, Bunworasate U, Goh YT, Ganly P, Huang H, et al. Chronic myeloid leukemia in the Asia-Pacific region: current practice, challenges and opportunities in the targeted therapy era. Leuk Res. 2010; 34:1459-71.

13. Jootar S. CML treatment in Asia-Pacific region. Hematology. 2012; 17 Suppl 1:S72-4.

14. Nakaseko C, Takahashi N, Ishizawa K, Kobayashi Y, Ohashi K, Nakagawa Y, et al. A phase 1/2 study of bosutinib in Japanese adults with Philadelphia chromosome-positive chronic myeloid leukemia. Int J Hematol. 2015; 101:154-64. 
15. Deininger MW, Kota V, Lipton JH, Milojkovic D, García Gutiérrez V, Leip E, et al. Bosutinib or imatinib in older vs younger patients with newly diagnosed chronic myeloid leukemia in the phase 3 BFORE trial. Blood. 2018; 132:1734-.

16. Brummendorf TH, Cortes JE, de Souza CA, Guilhot F, Duvillie L, Pavlov D, et al. Bosutinib versus imatinib in newly diagnosed chronic-phase chronic myeloid leukaemia: results from the 24-month follow-up of the BELA trial. Br J Haematol. 2015; 168:69-81.

17. Hsyu PH, Mould DR, Upton RN, Amantea M. Pharmacokinetic-pharmacodynamic relationship of bosutinib in patients with chronic phase chronic myeloid leukemia. Cancer Chemother Pharmacol. 2013; 71:209-18.

18. Mita A, Abumiya M, Miura M, Niioka T, Takahashi S, Yoshioka T, et al. Correlation of plasma concentration and adverse effects of bosutinib: standard dose or dose-escalation regimens of bosutinib treatment for patients with chronic myeloid leukemia. Exp Hematol Oncol. 2018; 7:9.

19. Kantarjian H, Shah NP, Hochhaus A, Cortes J, Shah S, Ayala M, et al. Dasatinib versus imatinib in newly diagnosed chronic-phase chronic myeloid leukemia. N Engl J Med. 2010; 362:2260-70.

20. Khoury HJ, Gambacorti-Passerini C, Brummendorf TH. Practical management of toxicities associated with bosutinib in patients with Philadelphia chromosome-positive chronic myeloid leukemia. Ann Oncol. 2018; 29:578-87.

21. Cortes JE, Apperley JF, DeAngelo DJ, Deininger MW, Kota VK, Rousselot P, et al. Management of adverse events associated with bosutinib treatment of chronic-phase chronic myeloid leukemia: expert panel review. J Hematol Oncol. 2018; 11:143. 
22. Kantarjian HM, Cortes JE, Kim DW, Khoury HJ, Brummendorf TH, Porkka K, et al. Bosutinib safety and management of toxicity in leukemia patients with resistance or intolerance to imatinib and other tyrosine kinase inhibitors. Blood. 2014; 123:1309-18.

23. Barber MC, Mauro MJ, Moslehi J. Cardiovascular care of patients with chronic myeloid leukemia (CML) on tyrosine kinase inhibitor (TKI) therapy. Hematology Am Soc Hematol Educ Program. 2017; 2017:110-4.

24. Medeiros BC, Possick J, Fradley M. Cardiovascular, pulmonary, and metabolic toxicities complicating tyrosine kinase inhibitor therapy in chronic myeloid leukemia: Strategies for monitoring, detecting, and managing. Blood Rev. 2018; 32:289-99.

25. Fujioka I, Takaku T, Iriyama N, Tokuhira M, Kimura Y, Sato E, et al. Features of vascular adverse events in Japanese patients with chronic myeloid leukemia treated with tyrosine kinase inhibitors: a retrospective study of the CML Cooperative Study Group database. Ann Hematol. 2018; 97:2081-8.

26. Fujisawa S, Nakamae H, Ogura M, Ishizawa K, Taniwaki M, Utsunomiya A, et al. Efficacy and safety of dasatinib versus imatinib in Japanese patients with newly diagnosed chronic-phase chronic myeloid leukemia (CML-CP): Subset analysis of the DASISION trial with 2-year follow-up. Int J Hematol. 2014; 99:141-53.

27. Nakamae H, Fujisawa S, Ogura M, Uchida T, Onishi Y, Taniwaki M, et al. Dasatinib versus imatinib in Japanese patients with newly diagnosed chronic phase chronic myeloid leukemia: a subanalysis of the DASISION 5-year final report. Int J Hematol. 2017; 105:792-804.

28. Nakamae H, Shibayama H, Kurokawa M, Fukuda T, Nakaseko C, Kanda Y, et al. Nilotinib as frontline therapy for patients with newly diagnosed $\mathrm{Ph}+$ chronic myeloid 
leukemia in chronic phase: results from the Japanese subgroup of ENESTnd. Int $\mathbf{J}$ Hematol. 2011; 93:624-32.

29. Nakamae H, Fukuda T, Nakaseko C, Kanda Y, Ohmine K, Ono T, et al. Nilotinib vs. imatinib in Japanese patients with newly diagnosed chronic myeloid leukemia in chronic phase: long-term follow-up of the Japanese subgroup of the randomized ENESTnd trial. Int J Hematol. 2018; 107:327-36.

30. Kizaki M, Takahashi N, Iriyama N, Okamoto S, Ono T, Usui N, et al. Efficacy and safety of tyrosine kinase inhibitors for newly diagnosed chronic-phase chronic myeloid leukemia over a 5-year period: results from the Japanese registry obtained by the New TARGET system. Int J Hematol. 2019; 109:426-39.

31. Goldberg SL, Savona M, Mauro MJ. Considerations for successful treatment-free remission in chronic myeloid leukemia. Clin Lymphoma Myeloma Leuk. 2018; 18:98105.

32. Rea D, Nicolini FE, Tulliez M, Guilhot F, Guilhot J, Guerci-Bresler A, et al. Discontinuation of dasatinib or nilotinib in chronic myeloid leukemia: interim analysis of the STOP 2G-TKI study. Blood. 2017; 129:846-54.

33. Ross DM, Masszi T, Gomez Casares MT, Hellmann A, Stentoft J, Conneally E, et al. Durable treatment-free remission in patients with chronic myeloid leukemia in chronic phase following frontline nilotinib: 96-week update of the ENESTfreedom study. $\mathbf{J}$ Cancer Res Clin Oncol. 2018; 144:945-54.

34. Mahon FX, Hughes TP. Treatment-free remission after second-line nilotinib treatment. Ann Intern Med. 2018; 169:510. 
35. Okada M, Imagawa J, Tanaka H, Nakamae H, Hino M, Murai K, et al. Final 3-year results of the dasatinib discontinuation trial in patients with chronic myeloid leukemia who received dasatinib as a second-line treatment. Clin Lymphoma Myeloma Leuk. $2018 ; 18: 353-60$ e1.

36. Saussele S, Richter J, Guilhot J, Gruber FX, Hjorth-Hansen H, Almeida A, et al. Discontinuation of tyrosine kinase inhibitor therapy in chronic myeloid leukaemia (EURO-SKI): a prespecified interim analysis of a prospective, multicentre, nonrandomised, trial. Lancet Oncol. 2018; 19:747-57. 
Table 1 Patient demographics and clinical characteristics

\begin{tabular}{lc}
\hline & Bosutinib $(N=60)$ \\
\hline Age, median (range), years & $55(20-83)$ \\
\hline Age group, $n(\%)$ & $41(68.3)$ \\
\hline$<65$ years & $19(31.7)$ \\
\hline$\geq 65$ years & $36(60.0)$ \\
\hline Sex, $n(\%)$ & $24(40.0)$ \\
\hline Male & $59.8(34.5-102.6)$ \\
\hline Female & $23.0(15.6-36.3)$ \\
\hline Weight, median (range), kg & $15(1-153)$ \\
\hline Body mass index, median (range), kg/m ${ }^{2}$ & $28(46.7)$ \\
\hline Time from CML diagnosis, median (range), days & $27(45.0)$ \\
\hline Prior CML therapy, ${ }^{a} n(\%)$ & $26(43.3)$ \\
\hline Sokal risk group, $n(\%)$ & $7(11.7)$ \\
\hline Low & \\
\hline Intermediate & $58(96.7)$ \\
\hline High & $2(3.3)$ \\
\hline 0 & \\
\hline & \\
\hline
\end{tabular}

${ }^{\text {aH} H y d r o x y u r e a ~(h y d r o x y c a r b a m i d e) ~ w i t h i n ~} 6$ months

$C M L$ chronic myeloid leukemia, ECOG Eastern Cooperative Oncology Group 
Table 2 Treatment summary

$$
\text { Bosutinib }(N=60)
$$

Duration of follow-up, median (range), months

$16.6(11.1-21.9)$

Duration of treatment, median (range), months

$15.3(0.3-21.9)$

Completed 12 months of treatment, ${ }^{\mathrm{a}} n(\%)$

$42(70.0)$

Discontinued treatment within 12 months, ${ }^{\mathrm{a}} n(\%)$

$18(30.0)$

Discontinued treatment, $n(\%)$

19 (31.7)

Due to adverse events

$18(30.0)$

Physician's decision

Dose reduction due to adverse events, $n(\%)$

400 to $300 \mathrm{mg}$ QD

300 to $200 \mathrm{mg}$ QD

8 (13.3)

Dose interruption due to adverse events, $n(\%)$

46 (76.7)

Dose escalation due to insufficient response, $n(\%)$
400 to $500 \mathrm{mg}$ QD
$6(10.0)$
500 to $600 \mathrm{mg}$ QD
$1(1.7)$

Dose intensity, median (range), mg/day

$354.7(95-494)$

${ }^{\mathrm{a}}$ Core treatment phase 
Table 3 Efficacy summary

$\%(90 \% \mathrm{CI}) \quad$ Bosutinib $(N=60)$

Primary endpoint

MMR at Month 12

$55.0(44.4-65.6)$

Secondary endpoints

MMR by Month 12

$61.7(51.3-72.0)$

CCyR by Month 12

$80.0(71.5-88.5)$

Exploratory endpoints

MMR at Month 3

$10.0(3.6-16.4)$

MMR at Month 6

50.0 (39.4-60.6)

MMR at Month 9

$53.3(42.7-63.9)$

$C C y R$ complete cytogenetic response, IS International Scale, $M M R$ major molecular response $(\leq 0.1 \% B C R-A B L 1$ on IS $)$ 
Table 4 Treatment-emergent adverse events ${ }^{\mathrm{a}}$

\begin{tabular}{|c|c|c|}
\hline \multirow[b]{2}{*}{$n(\%)$} & \multicolumn{2}{|c|}{ Bosutinib $(N=60)$} \\
\hline & Any grade & Grade 3/4 \\
\hline Any treatment-emergent adverse event & $60(100)$ & $45(75.0)$ \\
\hline Gastrointestinal & $52(86.7)$ & $9(15.0)$ \\
\hline Diarrhea & $52(86.7)$ & $9(15.0)$ \\
\hline Nausea & $17(28.3)$ & 0 \\
\hline Vomiting & $15(25.0)$ & $1(1.7)$ \\
\hline Constipation & $7(11.7)$ & 0 \\
\hline Upper abdominal pain & $6(10.0)$ & 0 \\
\hline Liver function & $48(80.0)$ & $29(48.3)$ \\
\hline Increased ALT & $33(55.0)$ & $20(33.3)$ \\
\hline Increased AST & $28(46.7)$ & $11(18.3)$ \\
\hline Increase blood alkaline phosphatase & $16(26.7)$ & 0 \\
\hline Liver disorder & $7(11.7)$ & $5(8.3)$ \\
\hline Hematologic & $27(45.0)$ & $16(26.7)$ \\
\hline Thrombocytopenia $^{\text {b }}$ & $18(30.0)$ & $5(8.3)$ \\
\hline Lymphopenia $^{\mathrm{c}}$ & $11(18.3)$ & $8(13.3)$ \\
\hline Neutropenia $^{\mathrm{d}}$ & $10(16.7)$ & $7(11.7)$ \\
\hline Anemia $^{\mathrm{e}}$ & $10(16.7)$ & 0 \\
\hline Leukopenia $^{\mathrm{f}}$ & $6(10.0)$ & $2(3.3)$ \\
\hline Infection & $39(65.0)$ & $4(6.7)$ \\
\hline Nasopharyngitis & $17(28.3)$ & 0 \\
\hline Upper respiratory tract infection & $6(10.0)$ & 0 \\
\hline Rash & $33(55.0)$ & $3(5.0)$ \\
\hline Rash & $16(26.7)$ & $1(1.7)$ \\
\hline Maculopapular rash & $8(13.3)$ & $1(1.7)$ \\
\hline \multicolumn{3}{|l|}{ Other } \\
\hline Increased lipase & $16(26.7)$ & $9(15.0)$ \\
\hline Pyrexia & $14(23.3)$ & $1(1.7)$ \\
\hline Increased GGT & $11(18.3)$ & $3(5.0)$ \\
\hline Increased amylase & $9(15.0)$ & $1(1.7)$ \\
\hline Back pain & $7(11.7)$ & 0 \\
\hline Headache & $7(11.7)$ & 0 \\
\hline
\end{tabular}




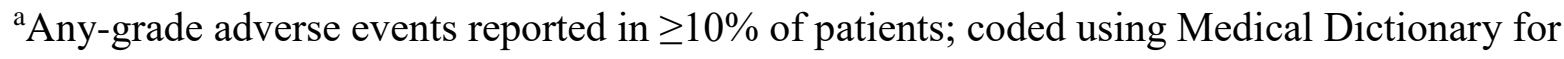
Regulatory Activities v21.1 terms and graded according to Common Terminology Criteria for Adverse Events v4.03

${ }^{\mathrm{b}}$ Clustered terms were thrombocytopenia and platelet count decreased

${ }^{\mathrm{c}}$ Clustered terms were lymphopenia and lymphocyte count decreased

${ }^{\mathrm{d}}$ Clustered terms were neutropenia and neutrophil count decreased

${ }^{\mathrm{e} C l u s t e r e d ~ t e r m s ~ w e r e ~ a n e m i a ~ a n d ~ h e m o g l o b i n ~ d e c r e a s e d ~}$

${ }^{\mathrm{f}}$ Clustered terms were leukopenia and white blood cell count decreased

$A L T$ alanine aminotransferase, $A S T$ aspartate aminotransferase, $G G T$ gamma-glutamyltransferase 
Table 5 Laboratory abnormalities ${ }^{\mathrm{a}}$

\begin{tabular}{lcc}
\hline & \multicolumn{2}{c}{ Bosutinib $(N=60)$} \\
\cline { 2 - 3 }$n(\%)$ & Any grade & Grade $3 / 4$ \\
\hline Any laboratory abnormality & $60(100)$ & $43(71.7)$ \\
Increased creatinine & $57(95.0)$ & 0 \\
Decreased lymphocyte count & $55(91.7)$ & $13(21.7)$ \\
Increased ALT & $51(85.0)$ & $29(48.3)$ \\
Increased AST & $49(81.7)$ & $16(26.7)$ \\
Decreased hemoglobin & $49(81.7)$ & $3(5.0)$ \\
Decreased platelet count & $38(63.3)$ & $6(10.0)$ \\
Decreased calcium & $37(62.7)$ & 0 \\
Increased alkaline phosphatase & $31(51.7)$ & 0 \\
Increased lipase & $28(46.7)$ & $13(1.7)$ \\
Decreased neutrophil count & $26(43.3)$ & $9(15.0)$ \\
Decreased albumin & $25(41.7)$ & 0 \\
Decreased white blood cell count & $23(38.3)$ & $2(3.3)$ \\
Decreased phosphate & $23(38.3)$ & $1(1.7)$ \\
Increased serum amylase & $21(35.0)$ & $1(1.7)$ \\
Increased creatine phosphokinase & $20(33.3)$ & $2(3.3)$ \\
Decreased potassium & $14(23.3)$ & $2(3.3)$ \\
Increased glucose & $13(21.7)$ & $2(3.3)$ \\
\hline
\end{tabular}

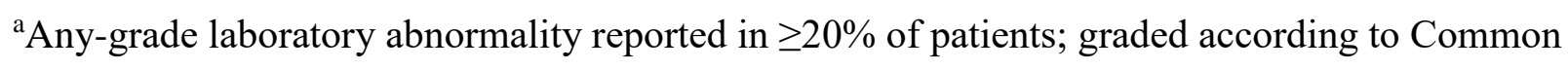

Terminology Criteria for Adverse Events v4.03

${ }^{\text {b59 }}$ patients evaluated for decreased calcium

$A L T$ alanine aminotransferase, $A S T$ aspartate aminotransferase 


\section{FIGURE LEGEND}

Fig. 1 Molecular response rates at Months 3, 6, 9, and 12

$I S$, International Scale; $M M R$, major molecular response ( $\leq 0.1 \% B C R-A B L 1$ on IS); $M R^{4}$, $\leq 0.01 \% B C R-A B L 1$ on IS; $M R^{4.5}, \leq 0.0032 \% B C R-A B L 1$ on IS 
Fig. 1 Molecular response rates at Months 3, 6, 9, and 12

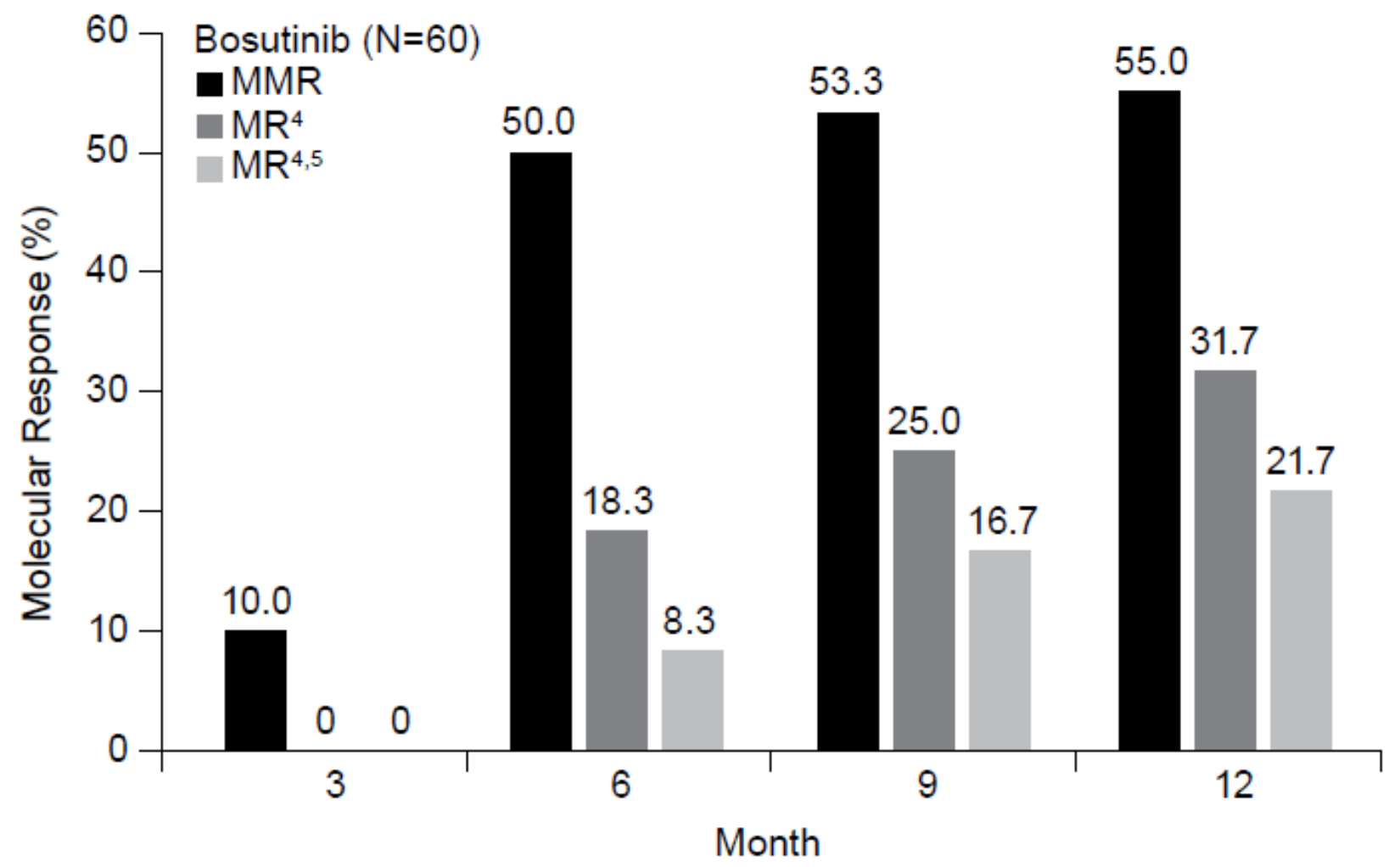

$I S$, International Scale; $M M R$, major molecular response $(\leq 0.1 \% B C R-A B L 1$ on IS $) ; M R^{4}$, $\leq 0.01 \% B C R-A B L 1$ on IS; $M R^{4.5}, \leq 0.0032 \% B C R-A B L 1$ on IS 


\section{SUPPLEMENTAL MATERIALS}

- Online Resource 1 Adverse events of special interest categories

- Online Resource 2 Time to molecular response, adjusting for competing risk of discontinuation without response

- Online Resource 3 Time to complete cytogenetic response, adjusting for competing risk of discontinuation without response

- Online Resource 4 Treatment-emergent adverse events of special interest

- Online Resource 5 Bosutinib trough concentrations

- Online Resource 6 Box plot of trough bosutinib plasma concentrations

- Online Resource 7 Bosutinib trough concentrations according to age group

- Online Resource 8 Box plot of trough bosutinib plasma concentrations according to age group 


\section{SUPPLEMENTAL MATERIALS}

International Journal of Hematology

Phase 2 study of bosutinib in Japanese patients with newly diagnosed chronic phase chronic myeloid leukemia

Masayuki Hino $^{1, *}$. Itaru Matsumura ${ }^{2} \cdot$ Shin Fujisawa $^{3} \cdot$ Kenichi Ishizawa $^{4}$ - Takaaki Ono ${ }^{5}$

Emiko Sakaida $^{6} \cdot$ Naohiro Sekiguchi $^{7} \cdot$ Yusuke Tanetsugu $^{8} \cdot$ Kei Fukuhara $^{8} \cdot$ Masayuki Ohkura $^{8}$

- Yuichiro Koide ${ }^{8} \cdot$ Naoto Takahashi ${ }^{9}$

${ }^{1}$ Osaka City University Hospital, Osaka, Japan

${ }^{2}$ Kindai University Hospital, Osaka, Japan

${ }^{3}$ Yokohama City University Medical Center, Yokohama, Japan

${ }^{4}$ Yamagata University Hospital, Yamagata, Japan

${ }^{5}$ Hamamatsu University Hospital, Shizuoka, Japan

${ }^{6}$ Chiba University Hospital, Chiba, Japan

${ }^{7}$ National Hospital Organization Disaster Medical Center, Tokyo, Japan

${ }^{8}$ Pfizer R\&D Japan G.K., Tokyo, Japan

${ }^{9}$ Akita University Hospital, Akita, Japan

\section{Contents}

Online Resource 1 Adverse events of special interest categories

Online Resource 2 Time to molecular response, adjusting for competing risk of discontinuation without response.... 3

Online Resource 3 Time to complete cytogenetic response, adjusting for competing risk of discontinuation without response

Online Resource 4 Treatment-emergent adverse events of special interest ....................................................6

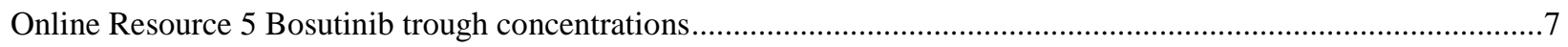

Online Resource 6 Box plot of trough bosutinib plasma concentrations ........................................................ 8

Online Resource 7 Bosutinib trough concentrations according to age group ....................................................

Online Resource 8 Box plot of trough bosutinib plasma concentrations according to age group ............................10

\footnotetext{
* Corresponding author at hinom@med.osaka-cu.ac.jp
} 
Online Resource 1 Adverse events of special interest categories

\begin{tabular}{|c|c|}
\hline Category & Adverse event criteria \\
\hline Cardiac & $\begin{array}{l}\text { - HLGTs in cardiac arrhythmias, heart failure, pericardial disorders } \\
\text { - PTs in cardiac death, sudden cardiac death, sudden death, ejection fraction decreased } \\
\text { - MedDRA SMQ (narrow): Torsade de pointes/QT prolongation }\end{array}$ \\
\hline Edema & - PTs contain edema, weight increased \\
\hline Effusion & - PTs in pleural effusion, pericardial effusion \\
\hline Gastrointestinal & $\begin{array}{l}\text { - PTs in nausea, regurgitation, retching, vomiting, vomiting projectile, diarrhea, defecation } \\
\text { urgency, frequent bowel movements, gastrointestinal hypermotility }\end{array}$ \\
\hline Hemorrhage & $\begin{array}{l}\text { - PTs in gastric occult blood positive, occult blood positive } \\
\text { - MedDRA SMQ (narrow): hemorrhage terms (excluding laboratory terms) }\end{array}$ \\
\hline Hypertension & $\begin{array}{l}\text { - HLGT in vascular hypertensive disorders } \\
\text { - PTs in BP abnormal, BP ambulatory abnormal, BP ambulatory increased, BP diastolic } \\
\text { abnormal, BP diastolic increased, BP increased, BP systolic abnormal, BP systolic } \\
\text { increased }\end{array}$ \\
\hline Infection & - SOC in infections and infestations \\
\hline Liver function & $\begin{array}{l}\text { - MedDRA (SMQ) hepatic disorders: sub-SMQs (narrow) in cholestasis and jaundice of } \\
\text { hepatic origin; hepatic failure, fibrosis and cirrhosis and other liver damage-related } \\
\text { conditions; hepatitis, noninfectious; liver-related investigations, signs and symptoms } \\
\text { (selected relevant) } \\
\text { - PTs: ALT abnormal, AST increased, AST abnormal, AST increased, bilirubin conjugated } \\
\text { abnormal, bilirubin conjugated increased, blood bilirubin abnormal, blood bilirubin } \\
\text { increased, blood bilirubin unconjugated increased, hepatic enzyme abnormal, hepatic } \\
\text { enzyme increased, hepatic function abnormal, hyperbilirubinemia, hypertransaminasemia, } \\
\text { liver function test abnormal, transaminases abnormal, transaminases increased, blood ALP } \\
\text { abnormal, blood ALP increased, liver function test increased }\end{array}$ \\
\hline Myelosuppression & $\begin{array}{l}\text { - MedDRA SMQs (narrow): hematopoietic cytopenias affecting >1 type of blood cell, }{ }^{\text {a }} \\
\text { hematopoietic erythropenia, hematopoietic leukopenia, hematopoietic thrombocytopenia } \\
\text { - PTs in bone marrow toxicity, hematocrit decreased, hemoglobin decreased, hematotoxicity, } \\
\text { anemia }\end{array}$ \\
\hline Rash & - HLTs in rashes, eruptions, and exanthem NEC; erythema; acne; dermatitis and eczema \\
\hline Renal & $\begin{array}{l}\text { - HLT in renal failure and impairment } \\
\text { - PTs in blood creatinine abnormal, blood creatinine increased, creatinine renal clearance } \\
\text { abnormal, creatinine renal clearance decreased, glomerular filtration rate abnormal, } \\
\text { glomerular filtration rate decreased }\end{array}$ \\
\hline Vascular & $\begin{array}{l}\text { - HLGTs in coronary artery disorders; arteriosclerosis, stenosis, vascular insufficiency and } \\
\text { necrosis; embolism and thrombosis } \\
\text { - HLTs in arterial therapeutic procedures (excluding aortic), CNS hemorrhages and } \\
\text { cerebrovascular accidents, CNS vascular disorders NEC, non-site specific vascular } \\
\text { disorders NEC, peripheral vascular disorders NEC (excluding the } 2 \text { PTs flushing and hot } \\
\text { flush), transient cerebrovascular events, vascular imaging procedures NEC, vascular } \\
\text { therapeutic procedures NEC }\end{array}$ \\
\hline
\end{tabular}

${ }^{a}$ The following MedDRA PTs were used for cytopenias: anemia, thrombocytopenia (thrombocytopenia, acquired amegakaryocytic thrombocytopenia), neutropenia (cyclic neutropenia, febrile neutropenia, idiopathic neutropenia, neutropenia)

$A L P$ alkaline phosphatase, $A L T$ alanine aminotransferase, $A S T$ alanine aminotransferase, $B P$ blood pressure, $C N S$ central nervous system, HLGT higher-level group term, HLT higher-level term, MedDRA Medical Dictionary for Regulatory Activities, NEC not elsewhere classified, $P T$ preferred term, $S M Q$ standardized MedDRA query, $S O C$ system organ class 
Online Resource 2 Time to first molecular response ${ }^{a}$, adjusting for competing risk of discentinuation without response

(a) MMR, (b) $\mathrm{MR}^{4}$, and (c) $\mathrm{MR}^{4.5}$

a.

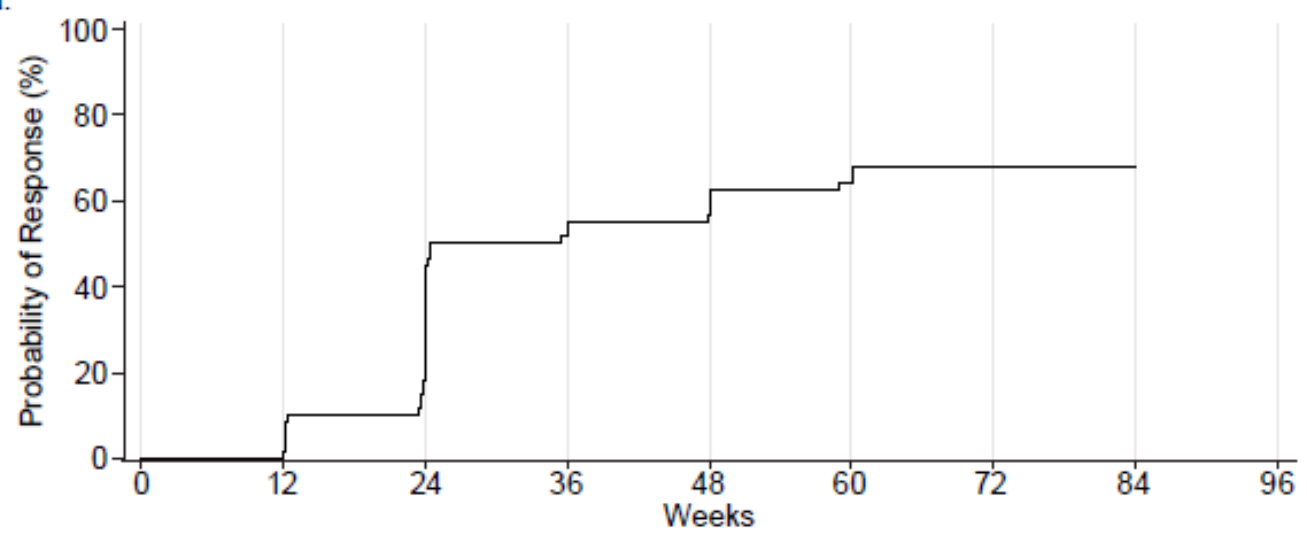

$\begin{array}{lllllllll}\text { No. at risk } & 60 & 56 & 35 & 13 & 8 & 4 & 2 & 2\end{array}$

b.

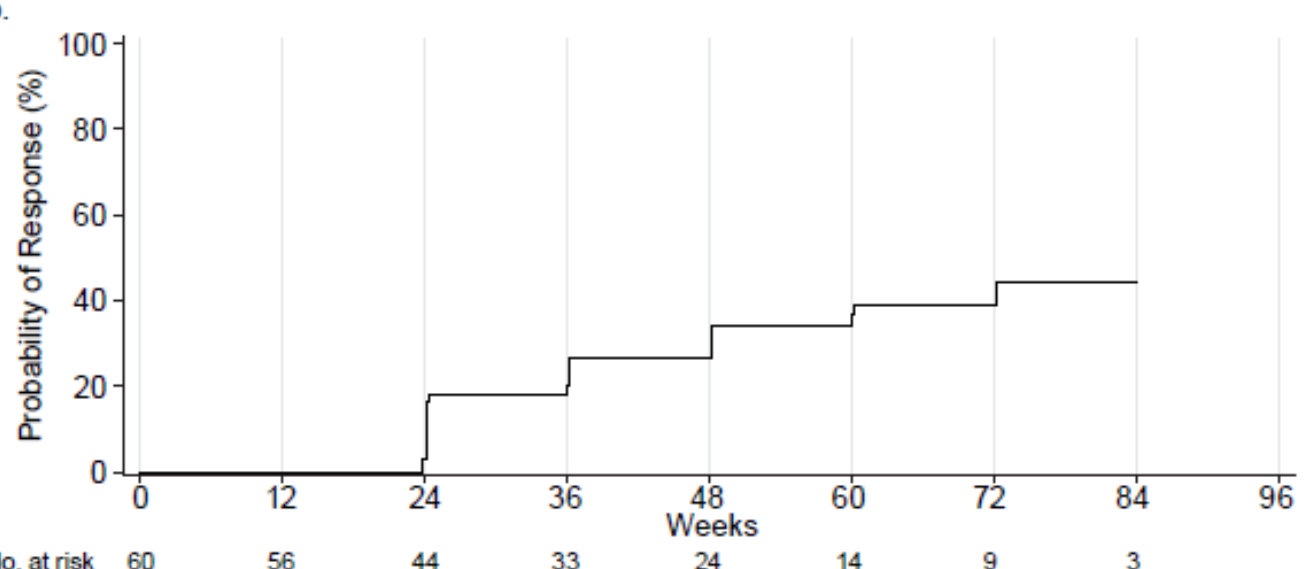

$\begin{array}{lllllllll}\text { No. at risk } & 60 & 56 & 44 & 33 & 24 & 14 & 9 & 3\end{array}$

c.

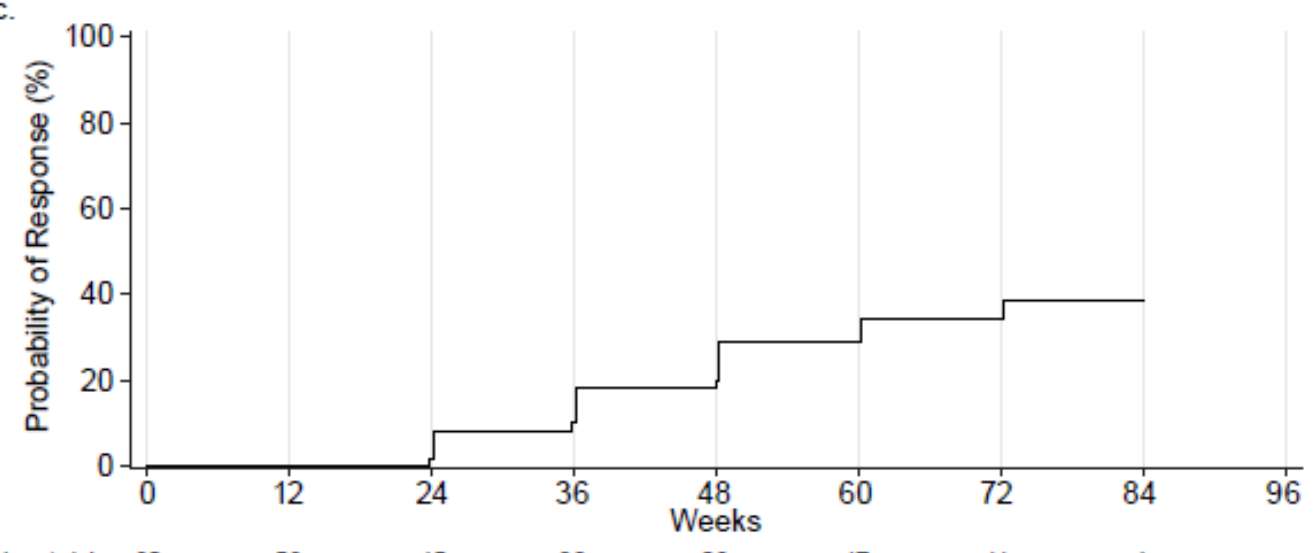

No. at risk $60 \quad 56$

45

38

29

$17 \quad 11$

4

${ }^{a}$ Molecular response was analyzed using cumulative incidence, adjusting for the competing risk without response.

IS International Scale, $M M R$ major molecular response ( $\leq 0.1 \% B C R-A B L 1$ on IS), $M R^{4} \leq 0.01 \% B C R$ $A B L 1$ on IS, $M R^{4.5} \leq 0.0032 \% B C R-A B L 1$ on IS 
Online Resource 3 Time to first complete cytogenetic response ${ }^{\text {a }}$, adjusting for competing risk of discontinuation without response

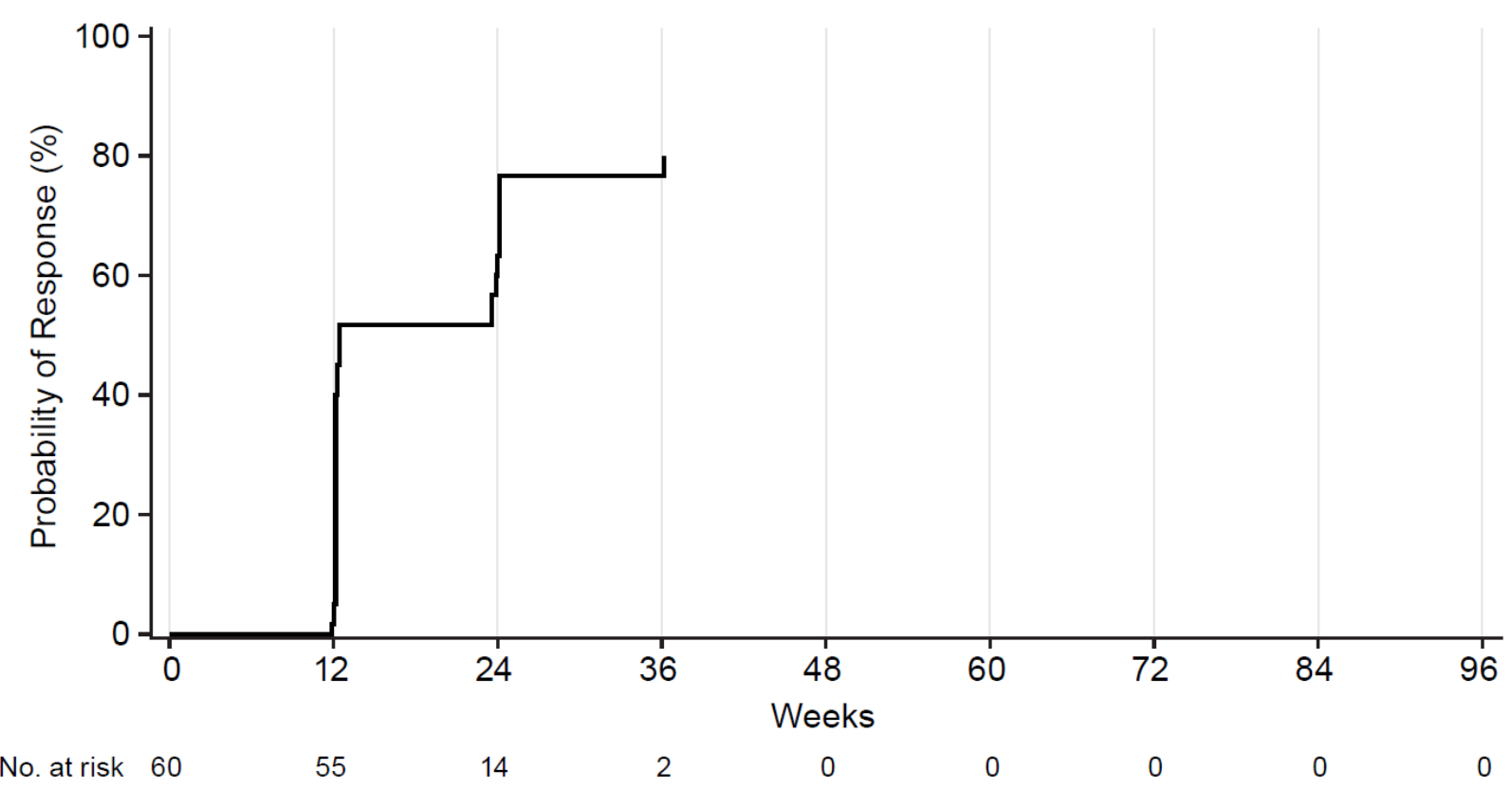

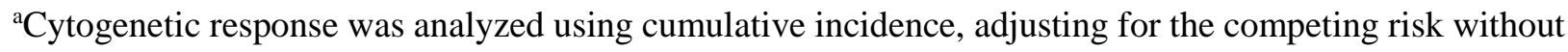
response. 
Online Resource 4 Treatment-emergent adverse events of special interest

\begin{tabular}{lcc}
\hline & \multicolumn{2}{c}{ Bosutinib $(N=60)$} \\
\hline Gastrointestinal & Any grade & Grade 3/4 \\
\hline Liver function & $52(86.7)$ & $9(15.0)$ \\
\hline Infection & $48(80.0)$ & $29(48.3)$ \\
\hline Rash & $39(65.0)$ & $4(6.7)$ \\
\hline Myelosuppression & $33(55.0)$ & $3(5.0)$ \\
\hline Effusion & $27(45.0)$ & $16(26.7)$ \\
\hline Hemorrhage & $5(8.3)$ & $1(1.7)$ \\
\hline Renal & $5(8.3)$ & 0 \\
\hline Cardiac & $4(6.7)$ & 0 \\
\hline Edema & $3(5.0)$ & 0 \\
\hline Hypertension & $3(5.0)$ & 0 \\
\hline Vascular & $1(1.7)$ & $1(1.7)$ \\
\hline
\end{tabular}


Online Resource 5 Bosutinib trough concentrations

\begin{tabular}{lc}
\hline Plasma levels, ng/mL & Bosutinib $(N=60)$ \\
\hline Day 28 & 34 \\
\hline$n$ & $83.6(64.1)$ \\
\hline Mean (SD) & $61.2-105.9$ \\
$95 \%$ CI & $66.5(1.38-363)$ \\
\hline Median (range) & 41 \\
\hline Day 56 & $86.0(46.4)$ \\
$n$ & $71.3-100.6$ \\
\hline Mean (SD) & $82.6(3.28-216)$ \\
\hline $95 \%$ CI & 44 \\
\hline Median (range) & $79.7(41.5)$ \\
\hline Day 84 & $67.0-92.3$ \\
\hline$n$ & $69.1(19.10-199)$ \\
\hline Mean (SD) & \\
\hline $95 \%$ CI & 51 \\
\hline Median (range) & $82.7(48.0)$ \\
\hline Average concentration ${ }^{\mathrm{a}}$ & $69.3-96.2$ \\
\hline$n$ & $71.2(7.59-260)$ \\
\hline Mean (SD) & \\
\hline $95 \%$ CI & \\
Median (range) & \\
\hline
\end{tabular}

${ }^{\mathrm{a}}$ Mean of Day 28, 56, and 84 trough concentrations

$C I$ confidence interval, $S D$ standard deviation 
Online Resource 6 Box plot of trough bosutinib plasma concentrations

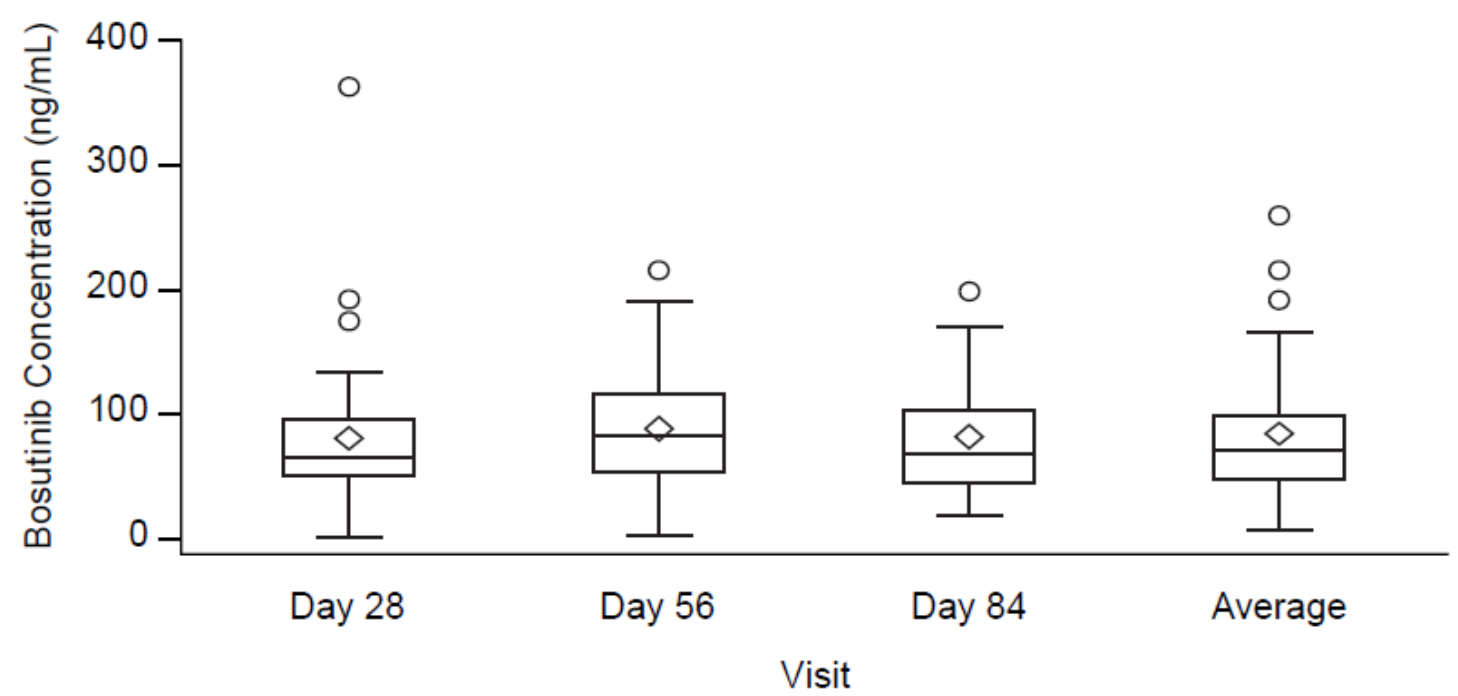

Box plot bounds represent $25 \%$ and $75 \%$ quartiles; inside the box, solid line represents median, and diamond represents mean; circles represent outliers and whiskers to the last points within 1.5 times the interquartile range. 
Online Resource 7 Bosutinib trough concentrations according to age group

\begin{tabular}{|c|c|c|}
\hline & \multicolumn{2}{|c|}{ Age group } \\
\hline Plasma levels, ng/mL & $\begin{array}{c}<65 \text { years } \\
(n=41)\end{array}$ & $\begin{array}{c}\geq 65 \text { years } \\
(n=19)\end{array}$ \\
\hline \multicolumn{3}{|l|}{ Day 28} \\
\hline$n$ & 25 & 9 \\
\hline Mean (SD) & $78.8(66.3)$ & 96.7 (58.9) \\
\hline $95 \% \mathrm{CI}$ & $51.4-106.2$ & $51.5-142.0$ \\
\hline Median (range) & $66.3(1.4-363.0)$ & $91.4(7.6-186.0)$ \\
\hline \multicolumn{3}{|l|}{ Day 56} \\
\hline$n$ & 33 & 8 \\
\hline Mean (SD) & $79.3(44.9)$ & $113.6(45.0)$ \\
\hline $95 \% \mathrm{CI}$ & $63.4-95.2$ & $76.0-151.1$ \\
\hline Median (range) & $72.5(3.3-216.0)$ & $108.5(40.7-191.0)$ \\
\hline \multicolumn{3}{|l|}{ Day 84} \\
\hline$n$ & 32 & 12 \\
\hline Mean (SD) & $73.4(36.4)$ & $96.3(51.0)$ \\
\hline $95 \% \mathrm{CI}$ & $60.3-86.5$ & $63.9-128.7$ \\
\hline Median (range) & $62.1(19.1-163.0)$ & $94.1(32.2-199.0)$ \\
\hline \multicolumn{3}{|l|}{ Average concentration ${ }^{\mathrm{a}}$} \\
\hline$n$ & 37 & 14 \\
\hline Mean (SD) & $80.3(47.5)$ & $89.3(50.4)$ \\
\hline $95 \% \mathrm{CI}$ & $64.4-96.1$ & $60.2-118.4$ \\
\hline Median (range) & $70.8(29.8-260.0)$ & $93.2(7.6-192.0)$ \\
\hline
\end{tabular}

${ }^{a}$ Mean of Day 28, 56 and 84 trough concentrations

$C I$ confidence interval, $S D$ standard deviation 
Online Resource 8 Box plot of trough bosutinib plasma concentrations according to age group

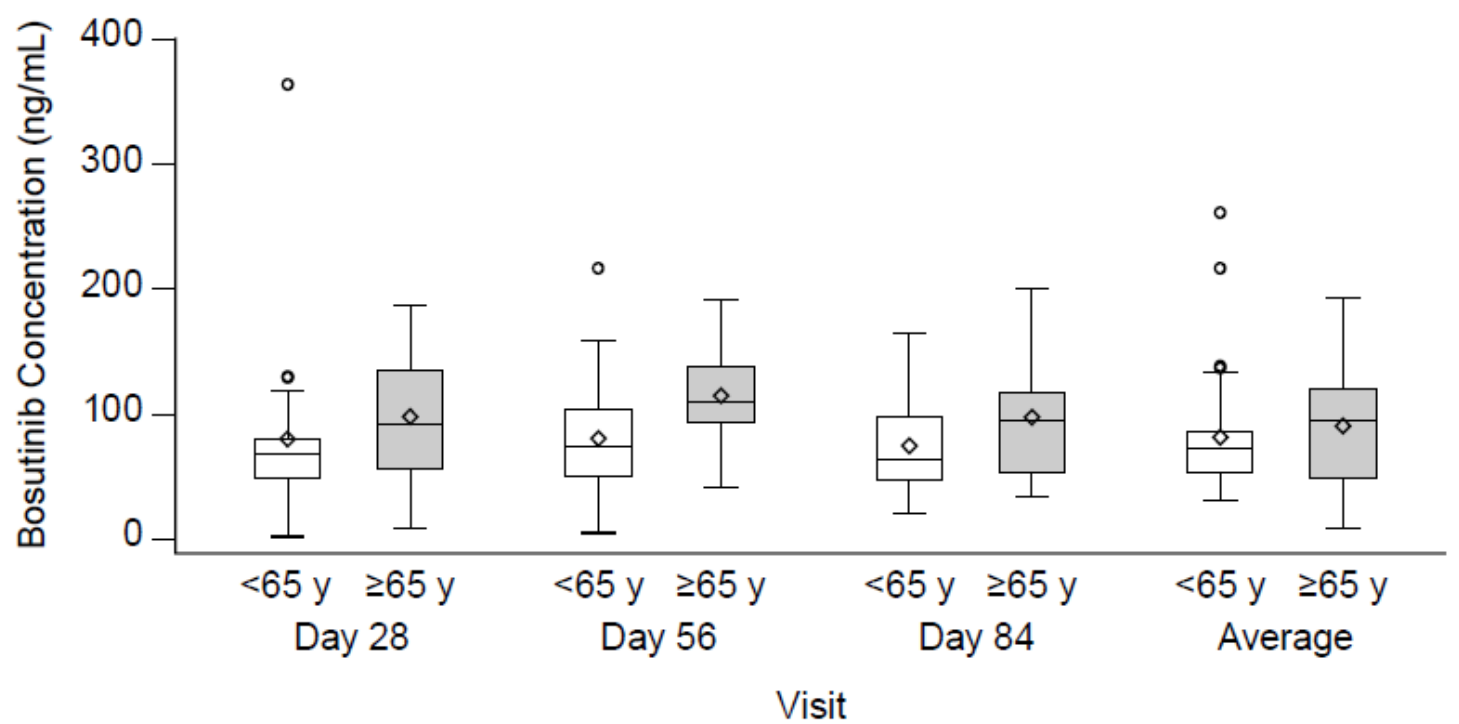

Box plot bounds represent $25 \%$ and $75 \%$ quartiles; inside the box, solid line represents median, and diamond represents mean; circles represent outliers and whiskers to the last points within 1.5 times the interquartile range. 